\title{
Identification of Relevant Images from Ancient Paintings using Statistical Distribution
}

\author{
G. G. Naidu' and Y. Srinivas ${ }^{2}$ \\ 'Department of Computer Engineering, Govt Polytechnic, Anakapalli, Visakhapatnam - 531001, \\ Andhra Pradesh, India; gagona.ggn@gmail.com \\ 2Department of Information Technology, GITAM University, Visakhapatnam - 530045, \\ Andhra Pradesh, India; sriteja.y@gmail.com
}

\begin{abstract}
Objectives: This article highlights a novel concept of identifying the ancient images along with the artists by proposing a model based on a Generalized Bivariate Laplacian Mixture Model (GBLMM) approach for this purpose. Methods/Statistical Analysis: Conservative Chinese paintings emulate the exquisiteness of Chinese sculpture. Most of these descriptions are digitized and are available in the internet. One of challenging task is to retrieve the images of significance more efficiently and proficiently. This article highlights a pioneering idea to spot these ancient images along with the artist by proposing a model based on a Generalized Bivariate Laplacian Mixture Model (GBLMM) approach. Findings: The methodology is subjected to the application of ancient Chinese paintings. The results derived are evaluated against quality metrics such as image fidelity, peak signal noise ratio, structured coefficients, average difference and mean squared error. The proposed model accomplished over $97 \%$ of classification accuracy over a dataset containing 3750 traditional Chinese paintings. Application/Improvements: In order to ascertain the quality of segmentation, metrics like Image Fidelity (IF), Mean Squared Error (MSE) and Peak Signal Noise Ratio (PSNR) are considered. This model can be very much useful for the archeologists to identify the ancient paintings.
\end{abstract}

Keywords: Ancient Paintings, GBLMM, Mixture Model, Quality Metrics, Retrieval

\section{Introduction}

Image processing aims at development of algorithms for better understanding of the image details, ability to analyze the details, enhancing the images and retrieval of significant regions of interest from these images. The recent developments in this area help to safe guard the vital paintings available in the archeological departments. Many historic paintings and literature relevant to the ancient paintings is available in the archeological departments.

It is therefore necessary to retain this information so that the glory of the paintings together with the sculptures, artists and the period to which their paintings belongs can be estimated. Versatile models are developed by several researchers based on methods like: Identifying the regions of interests, identifying the regions based on the strokes, the period of the painting together with the artists' details can be easily identified ${ }^{1,2}$. Analysis of literature mostly highlighted the techniques based on approaches based on consideration of stroke, attribute mining, autograph, color amalgamation and speckles ${ }^{3}$. Next level developments are also spotted in the survey literature, which signifies the concepts of Data miming. Amid these methods, classification and clustering techniques are utilized numerously.

Models based on other approaches like Statistical Model Based Techniques, Artificial Neural Networks, Artificial Intelligence Techniques, Template Based Approaches are also enlightened in literature. Among the

${ }^{*}$ Author for correspondence 
above presented methodologies, classifications of images based on the attributes are mostly preferred. Yet, during the classification and recognition of painting images, the disparity among the colors, the distinction among the pixels, concentration of strokes, orientation are given minimal weight.

In view of the fact, that model based approaches assure effectual results ${ }^{3}$ to have effective classification results, methodologies are hence projected using statistical distributions. To have more clear-cut results, the parameters within every region of the image need to be updated. The Expectation Maximization (EM) algorithm proposed ${ }^{4}$ is therefore considered for this very purpose. The EM algorithm facilitates to optimize the parameter set and the entire retrieval process is based on these obtained updated parameters.

The current literature in the field of image classification and identification of images is presented in this section, highlighted about the significance of the attribute descriptors together with their usage ${ }^{5}$, Contour shape descriptors can be of more significance. Among the widely used descriptors for image retrievals, the authors have also noticed that Curvature Scale Space Descriptors (CSSD) together with Fourier Descriptors (FD) will be effective. The impact and significance of curvature scale space descriptors are also discussed. However, no broad evaluation has been made amid these two shape descriptors.

Query Feedback for Interactive Image Retrieval have highlighted the need for understanding the visual content in multimedia during indexing and retrieval and also accentuated the need for online interactive learning ${ }^{6}$. Also highlighted that ability and speed are imperative factors in interactive visual content retrieval, while most of the existing methods insist on unendorsed assumptions on likeness reckoning and learning algorithms. Particularly, content-based image retrieval techniques normally suppose that perceptually identical images are positioned close to each other within a correlated region.

The new technologies coming up in order to capture and maintain digital imagery ${ }^{7}$. The authors confer a number of core strides in the recent decade associated to image reclamation and computerized image annotation and also discussed the difficulties associated with at present used mechanisms targeted towards effective image retrieval. The need for newer technologies in particular to capture TCP images and stressed the importance of knowledge while retrieving the system in order to browse a particular image of interest ${ }^{8}$. The authors have considered Gongbiand Xieyi, the traditional Chinese paintings and highlighted the need for methodologies for effective classification of these images. The authors have considered the Support Vector Machine.

In content-based image retrieval systems, studies focused on shifting from scheming complicated low-level attribute mining algorithms to semantic methods?. The authors surveyed of the current technical attainments in high-level semantic-based image retrieval. Identified five categories of the state-of-the-art techniques: 1. Object ontology, 2. Machine learning methods, 3. Relevance feedback, 4. Semantic template, 5. Fusing.

Image Processing for artist identification, understanding of the artist common practices, scrupulous comparisons of an array of technical data and visual evaluation of the artist's hand writing ${ }^{10}$. The authors highlighted to brush work analysis and artist recognition. Fusion of several biometrics for human validation, performance and improvement has received combining facial and iris based traits using score level fusion ${ }^{11}$. Fusion rule considered for identifying the matching scores.

Classification of Ancient Manuscripts, categorization of ancient handwritten documents according to the characters style ${ }^{12}$, taken into account a sample set of features derived from the hand written images based on the shape of the handwritten patterns. The impression strokes along with the orientation are plotted and the corresponding histograms are extracted at a comprehensive level from confined contour annotations. The investigations and importance of the feedback mechanisms in a multimedia structure planned for the interactive exploration and explanation of creative collections ${ }^{13}$, experimented using diverse techniques like mean shift feature distortion algorithm, standard relevancy feedback mechanisms and learning based techniques. While evaluating the tests they used co-variance matrices in order to achieve effective likeness retrieval upon images.

Knowledge discovery of artistic influences focused on the comparison between the painted images, with the visual similarity ${ }^{14}$. This assessment is based on computer vision approaches and machine learning. An accurate and capable image identification mining model, by taking hierarchical k-Means algorithm ${ }^{15}$, signified the usage of data mining concepts into the image domain. They highlighted the applications of data mining concepts in areas 
like computer vision, image processing, image retrieval, machine learning and artificial intelligence. They highlighted two different clustering algorithms namely, hierarchical and k-Means.

Methodologies based on content based image retrievals are also projected in the literature, where the relevant images of interest can be retrieved by a query. However, most of these techniques are based on low level attribute extraction and therefore these models failed to retrieve images having high level features. Semantic based techniques are also developed to identify the images based on the content and semantic interpretation ${ }^{16}$. However, these methodologies also could not give the expected results.

Model based techniques for classification and retrieval of ancient paintings is also proposed ${ }^{17}$. In the presented article, a tag is associated for every image, which acts as a feature and thereby the retrieval or detection of an image is wholly based on these tags. In this article, the Meta tags and the color of the image are taken as features. These features are given as inputs to the Generalized Bivariate Laplacian Mixture Model (GBLMM) for the retrieval of relevant images and the identification of the artist together with the period of painting. In order to put into practice, the Chinese data set of considered.

The rest of the article is structured as follows. In Section 2, deals with the GBLMM. The significant challenges that hinder the accuracy are showcased in Section 3 and the data set considered is presented in Section 4. Section 5 of the paper highlights the features selection methodology. The results derived together with performance evaluation models are summarized in the Section 6.

\section{Generalized Bivariate Laplacian Mixture Model}

The Probability Density Function (PDF) of GBLMM is given as:

$$
\begin{aligned}
f(x, y)= & \frac{1}{\pi \sigma_{1} \sigma_{2} \sqrt{\left(1-\rho^{2}\right)}} \\
& k_{0} \sqrt{\left.\left(2\left(x^{2} / \sigma_{1}^{2}-2 p x y\right) /\left(\sigma_{1} \sigma_{2}\right)+\frac{y^{2}}{\sigma_{2}^{2}}\right) /\left(1-\rho^{2}\right)\right)}
\end{aligned}
$$

Where $k_{0}$ the Bessel function is $p$ is scale parameter, $0 \leq$ $p \leq 1$
The main intuition to identify the specks within every image region, GBLMM is considered. In this procedure, every image under consideration is alienated into four parts, the first two parts pointing the least significant bits and the next two parts for identification of the high frequency spectrums. These parts, for convention named as short range spectrums, moderate range spectrums, partially high range spectrums and very high range spectrums. This division is based on the consideration of discrete wavelet Haar. Low range spectrums are considered for estimating the small variations within the image regions, along the horizontal side, medium spectrum bands help in recognizing the change along the vertical side and High frequency spectrums help in estimating the variations within the image regions having higher degree of deviations. For each of the spectrums, the histograms are plotted. The pixels are grouped into clusters based on the histogram range and each pixels insider every group are modeled using the proposed model based on GBLMM and thereby significant PDF's are recognized. This is an iterative process; where for further reduction in space can be planned by repeating the above procedure further reduction of space can be possible, where the high band spectrums are given as inputs to the model and estimating the relevant PDFs. KL-divergence is considered for identifying the effectiveness between the retrieved images against the trained images. Finally the paper is verified against quality metrics for detection of the similarity.

\section{Major Challenges}

A good number of the articles in the area of ancient painting retrievals and identification are subjected to degenerate algorithms such as edge based, region based, intensity based, stroke based and machine learning approaches together with soft computing techniques. However, in most cases, when dealing with the identification of sculptures and painting which are ancient, it is customary to identify the inherent parameters which help for effective recognition. Therefore, model based techniques are more appropriate in this direction. However, among the statistical models, GBLMM is considered in this article because of its ability to recognize the ancient images, as it contains long tails and using these tails, the patterns of the pixels can be more robustly analyzed. Also it has the capacity to link the relevant pixels more appropriately which is major consent while identification of images. 


\section{Dataset Considered}

In order to present the model, the databases available in the internet of Chinese images are considered. In particular, the image considered by C.C. Chang, et. al. (1996) is also considered. The lists of images considered are presented in Figure 1.

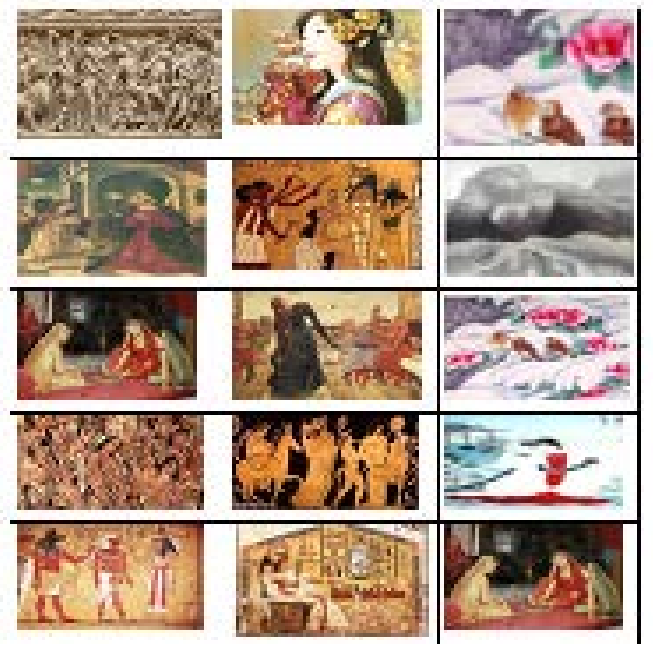

Figure 1. The image dataset.

\section{Features Selection and Methodology}

In order to retrieve the relevant information between images, we have considered two features, namely the stroke and the color. These features are considered as input to the model presented in Section 3 of the paper. Each one of the images from the considered dataset is taken into account and the relevant attributes are identified. These features are mapped to the model for extracting the likelihood estimates (PDF). Each of the images will have a set of PDF's. In order to recognize the similar paintings, the relevancy between the PDF's is to be considered. For this purpose KL-divergence or Image similarity methods based on equalitarian distance will be more appropriate. In this article, we have considered the relevance feedback mechanism for ensuring the relevancy among the images.

\section{Results}

The segmentation process for every image is carried out using the GBLMM. Against every pixel of the image region, the PDF values are attained. The image under consideration is segmented and the appropriate values of the PDF's are identified. The image reconstruction is based on scribing the colors to each of the pixel distributed among the clusters. In the present work, we have taken color image, identified the gray level equivalent and corresponding segmented image are obtained. In order to ascertain the quality of segmentation, metrics like Image Fidelity (IF), Mean Squared Error (MSE) and Peak Signal Noise Ratio (PSNR) are considered in the corresponding values are tabulated in Table 1.

Table 1. Performance Metrics - MSE, SNR, IF

\begin{tabular}{|c|c|c|c|c|}
\hline \multirow{2}{*}{ Image } & \multirow{2}{*}{$\begin{array}{l}\text { Segmented } \\
\text { output }\end{array}$} & \multicolumn{3}{|c|}{ Evaluation Metrics } \\
\hline & & MSE & PSNR & IF \\
\hline (ang) & 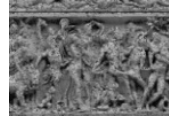 & 0.567 & 32.7 & 0.73 \\
\hline & & 0.40 & 38.9 & 0.67 \\
\hline & & 0.37 & 43.7 & 0.67 \\
\hline & & 0.447 & 58.74 & 0.74 \\
\hline & & 0.219 & 68.6 & 0.52 \\
\hline & & 0.138 & 52.74 & 0.81 \\
\hline & & 0.271 & 51.62 & 0.38 \\
\hline
\end{tabular}

\section{Conclusions}

This article highlights a novel contribution for proper identification of the images. The proposed model is tested for accuracy using performance evaluation metrics and the proposed methodology has showcased better recognition accuracy. This model can be very much useful for the archeologists to identify the ancient paintings.

\section{References}

1. Chen CC, Bimbo AD, Amato G, Boujemaa N, Outhemy P, Kittler J, Pitas I, Smeulders A, Alexander K, Kiernan 
K, Li CS, Wactlar H, Wang JZ. Report of the DELOS-NSF Working Group on Digital Imagery for Significant Cultural and Historical Materials. 2002.

2. Bouman C, Liu B. Multiple resolution segmentation of textured images. IEEE Transactions on Pattern Analysis and Machine Intelligence. 1991; 13(2):99-113. crossref

3. Pal SK, Pal NR. A review on image segmentation techniques. Pattern Recognition. 1993; 26(9):1277-94. crossref

4. McLachlan G, Krishnan T. The EM algorithm and extensions. New York: John Wiley and Sons; 1996. p. 1-304. PMid: 8568455

5. Zhang D, Lu G. A comparative study of curvature scale space and Fourier descriptors for shape-based image retrieval. Journal of Visual Communication and Image Representation. 2003; 14(1):39-57. crossref

6. Kushki A, Androutsos P, Plataniotis KN, Venetsanopoulos AN. Query feedback for interactive image retrieval. IEEE Transactions on Circuits and Systems for Video Technology. 2004; 14(5):644-55. crossref

7. Datta R, James JL, Wang Z. Content-based image retrieval - Approaches and trends of the new age. Proceedings of the 7th ACM SIGMM International Workshop on Multimedia Information Retrieval; 2005. p. 253-62.

8. Jiang S, Huang Q. An effective method to detect and categorize digitized traditional Chinese paintings. Pattern Recognition Letters. 2006; 27(7):734-46. crossref

9. Liu Y, Zhang D, Lu G, Ma WY. A survey of contentbased image retrieval with high-level semantics. Pattern Recognition. 2006; 40(1):262-82. crossref
10. Johnson CR, Hendriks E. Image processing for artist identification. IEEE Signal Processing Magazine. 2008; 25(4):3748. crossref

11. Wang F, Han J. Multimodal biometric authentication based on score level fusion using Support Vector Machine. OptoElectronics Review. 2009; 17(1):59-64. crossref

12. Siddiqi I, Cloppet F, Vincent N. Contour based features for the classification of ancient manuscripts. Proceedings of the 14th Conference of the International Graphonomics Society; 2009. p. 226-9.

13. Grana C, Borghesani D, Cucchiara R. Relevance feedback strategies for artistic image collections tagging. Proceedings of the 1st International Conference on Multimedia Retrieval, ICMR; 2011. crossref

14. Saleh B, Abe K, Elgammal A. Knowledge discovery of artistic influences: A metric learning approach. The Fifth International Conference on Computational Creativity; 2014.

15. Dhonde P, Raut CM. Precise and proficient image mining using hierarchical K-Means algorithm. International Journal of Scientific and Research Publications. 2015; 5(1):1-4.

16. Padala A, Yarramalle S. Krishna Prasad MHM. An approach for effective image retrievals based on semantic tagging and generalized gaussian mixture model. I.J. Information Engineering and Electronic Business. 2015; 3:39-44. crossref

17. Naidu GG, Srinivas Y. Model based approach for identification of relevant images from ancient paintings. International Journal of Engineering and Manufacturing (IJEM). 2017; 7(5):39-47. crossref 